\title{
Geometric triangular chiral hexagon complexes and clonal embryogenic body organization on the Turin Shroud crucified man image: A predictable tissue response to injury
}

\author{
Jairo A. Diaz \\ Department of Pathology, Medicine School, Laboratory of Pathology, Hospital Departmental of Villavicencio, Hospital Departmen- \\ tal of Granada, University Cooperative of Colombia, Villavicencio, Colombia; jaditod@hotmail.com
}

Received 15 July 2013; revised 15 August 2013; accepted 24 August 2013

Copyright (c) 2013 Jairo A. Diaz. This is an open access article distributed under the Creative Commons Attribution License, which permits unrestricted use, distribution, and reproduction in any medium, provided the original work is properly cited.

\section{ABSTRACT}

The shroud continues to remain one of the most studied and controversial artifacts in human history. Many tests, X-ray fluorescence, reflectance, spectrometry and low energy/high-resolution $X$ ray transmission have shown that the crucified body is not compatible with a painted image. Researchers confirm that the alleged blood is real blood. We documented the self-assembly of geometric triangular chiral hexagon complex (GTCHC) with structural organization of embryoid bodies in cancer tissues. The identification of these structures is not only limited to malignant tumors but also appears in extreme injured tissues. Our interest is to determine if we can predict and identify these patterns in the Shroud of Turin. Based on pattern recognition image was analyzed over 100 shroud images. We identified a central spectral emission line that exhibits a characteristic signature on a plot of residual electromagnetic radiation, head area narrowing and low extremities broadening, indication of decay energy changes in the velocity of the molecules in the traversal trajectory. This Electromagnetic collision event generates in the cloth stagnant blood areas with patterns identical to those identified for us in cancer damage tissues. Inflammatory cytokines activate stem cells and Notch signaling proteins in cascade of interactions to generate real clonal human embryoid template. Can we predict function from structure? These structures evoke life, regeneration, but not death. Our findings suggest the image of a crucified man on the Shroud of Turin is a real physical electromagnetic collision event in response to extreme tissue injury, with the fact that supports our previous findings in cancer tissues as real and predictable. Proteins derived from these emergent damage tissue derivate stem cells could be used to design biologic templates in regenerative medicine and develop novel strategies in cancer therapy.

Keywords: Turin; Shroud; Geometric; Triangular; Chiral; Hexagon; Embryogenic

\section{INTRODUCTION}

The shroud of Turin is probably the most important relic of the Christianity, and a real observable sacred linen that connects a theology doctrine with a terrestrial material symbol with more than 2000 years of antiquity. The shroud continues to remain one of the most studied and controversial artifacts in human history. No one has a good idea about how front and back images of a crucified man came to be on the cloth. Yes, it is possible to create images that look similar. But no one has created images that match the chemistry, peculiar superficiality and profoundly mysterious three-dimensional information content of the images on the Shroud. In 1988 a sample cut from the shroud was radiocarbon dated to indicating a cloth source date ranging between 1260 and 1390 [1]. Many shroud researchers today believe the sample tested was not representative of the entire shroud [2]. And that historical data now reliable place the shroud in Constantinople in 1204 well before the date indicated by carbon dating. Many tests including X-ray fluorescence, reflectance, spectrometry and low energy/high-resolution $\mathrm{X}$-ray transmission have shown that the body image is 
not compatible with a painted image [3-12]. In 1980 biophysicist John Heller and chemist Allan Adler collaborated in publishing a report on chemical testing, they conducted on Shroud sticky tape samples. Their research confirms that the alleged blood on the shroud is real blood [13]. In 1981 Heller and Adler extended their research of blood shroud samples to include serological techniques, which involve the diagnostic identification of two major blood serum proteins: Albumin and immunoglobulin antibody. This research was able to further classify the blood on the shroud as primate blood which is most likely of human origin [14]. In 1984 Italian professor Balma Ballone pathologist using serological techniques confirmed the identification of immunoglobulin in shroud blood, and he also evaluated the expression of additional blood components specifically the antigens identified as $\mathrm{M}, \mathrm{N}$, $\mathrm{S}$. The research shows that the blood on the Shroud is $\mathrm{M}$, $\mathrm{N}, \mathrm{S}$ positive. Unlike $\mathrm{M}$ and $\mathrm{N}$ antigens which are shared between certain primates and humans, the $\mathrm{S}$ antigen is exclusive to humans only. The positive result indicates human blood [15].

From another complementary point of view, we documented how intercellular cancer collision events generate geometry. We described and documented the selfassembly of geometric triangular chiral hexagon crystallike complex organizations (GTCHC) in human pathologic tissues. We found this architectural geometric expression at macroscopic and microscopic levels, mainly in cancer processes [16]. In our experimental model, we were able to reproduce and predict polarity, chirality, helicoid geometry, and triangular and hexagonal clusters through electromagnetic sequential collisions. In addition, we also determined that similar events among the constituents of extracellular matrix, which drive and produce piezoelectric activities, are responsible for the genesis of GTCHC complexes in pathological tissues. This study suggests that molecular crystals, represented by triangular chiral hexagons derived from a collision-attraction event against collagen type 1 fibrils, emerge at microscopic and macroscopic scales, presenting a lateral assembly of each side of hypertrophy helicoid fibers that represent energy flow in cooperative hierarchically chiral electromagnetic interaction in pathological tissues, and arise as a geometry of the equilibrium in perturbed biological systems. Additionally, we also documented and gathered insights into the magnetic field (MF) in cancer tissues and how it generates a functional geometric attractor complex in their entangled state [17]. This geometry occurs on documented collider partners, which are pairs of spiral sub patterns twisted in opposite directions, generating powerful electromagnetic forces during this rotational movement. These forces are exerted over collagen type 1 fibrils and influence the dipole behavior of vascular cells. Three very fundamental sequential steps are integrate: Initiation phase begins from the moment at which thousands of molecular biologic crystals, represented by triangular chiral hexagons derived from collision attraction events against collagen type I fibrils, emerge at microscopic and macroscopic levels. This phase is full of geometric complexes. The essential basis of this geometry is the triangulation and generation of mirror images that conclude in hexagons. Generator phase electromagnetic forces are dominant in forming biologic molecules. Colliding pairs of spirals that are oriented in opposite directions generate powerful electromagnetic fields. Fractals represent the echo image of crystal growth vibration generated by the spiral rotation of electromagnetic waves, and are the sum of the total fractal resonances, which are the convergent and confluent phase of the system. Differentiation embryoid bodies (Figures 1-4) represent the final expression of this convergent flow of information through triangular chiral crystal molecules (i.e., GTCHC) and assembly in a light optic polarized electromagnetic corridor.

Fractal geometry makes spatial correction errors embrace the chaotic system in a way that permits new structures to emerge, and as a result, an ordered self-assembly of embryoid bodies with neural differentiation at the final stage of cancer development is a predictable process [18, 19] (Figure 5).

We observed a highly immunostain-selective distribution of the neural enolase antibody related to clusters of template triangular chiral mirror-differentiated malignant
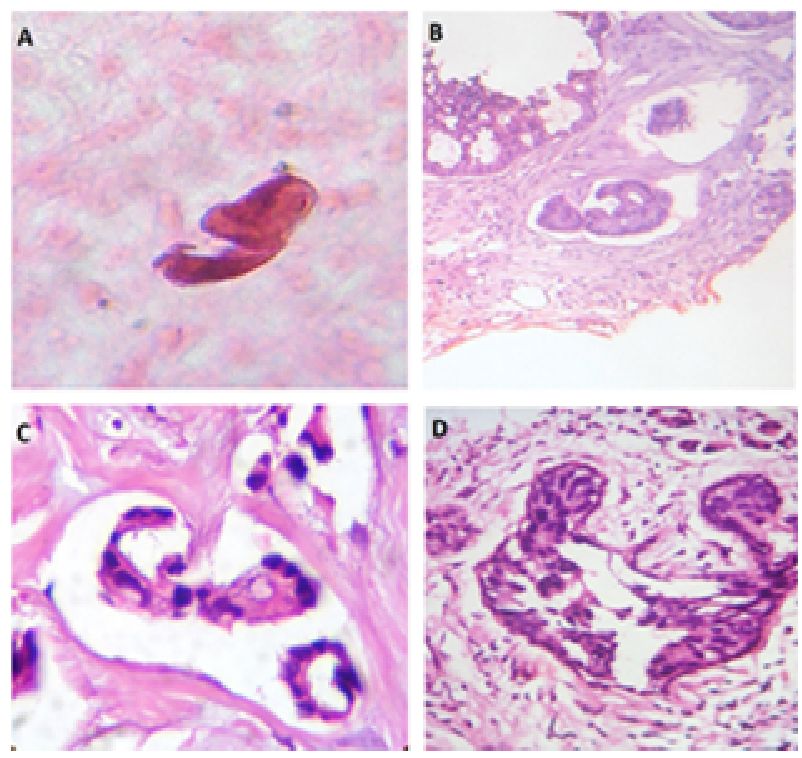

Figure 1. Identification of embryogenic bodies in cancer tissues. A shows embryogenic body in smear cytology from peritoneal carcinomatosis. Ascitic fluid Papanicolaou stains 40×. B illustrates embryogenic pattern identified in breast adenocarcinoma $\mathrm{H}$ E stain $40 \times$. C illusttates embryogenic body in colon adenocarcinoma $\mathrm{H}$ E stain $40 \times$. D shows embryogenic pair bodies in prostate adenocarcinoma. H E stain $40 \times$. 

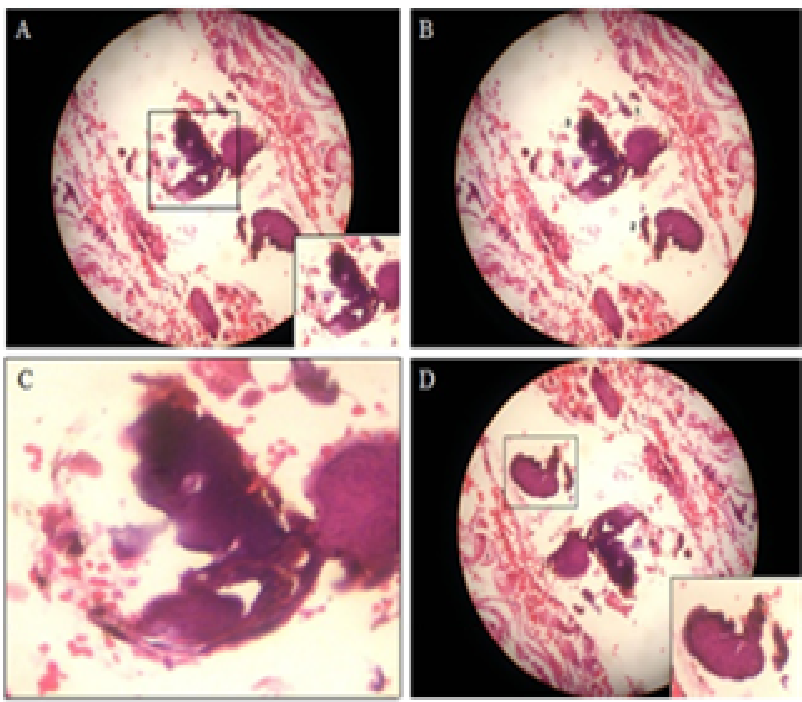

Figure 2. A, B, C, D shows embryogenic pattern identified in sarcoma tumor. H E stain $40 \times$.


Figure 3. A: it illustrates a gross surgical sample of tail pancreas cancer. B: it shows a macro vacuole where lies an embryogenic body which is point out. C: it displays a closed up of the embryonic body found in the surgical sample.

cells organized in an embryoid body phenotype assemblage. There was positive antibody activity in relation to the chiral pattern organization of groups of cells in the context of the restricted area of the tumor. Thus, enolase can be used to track and facilitate their identification (Figure 6).

Our latest observations indicate that the presence of these embryogenic bodies is not only limited to tumor tissues, but also appears naturally in damage tissues by traumatic injuries (Figure 7).

Also we document how electromagnetic fields released in collision-impact events generates fractal in-

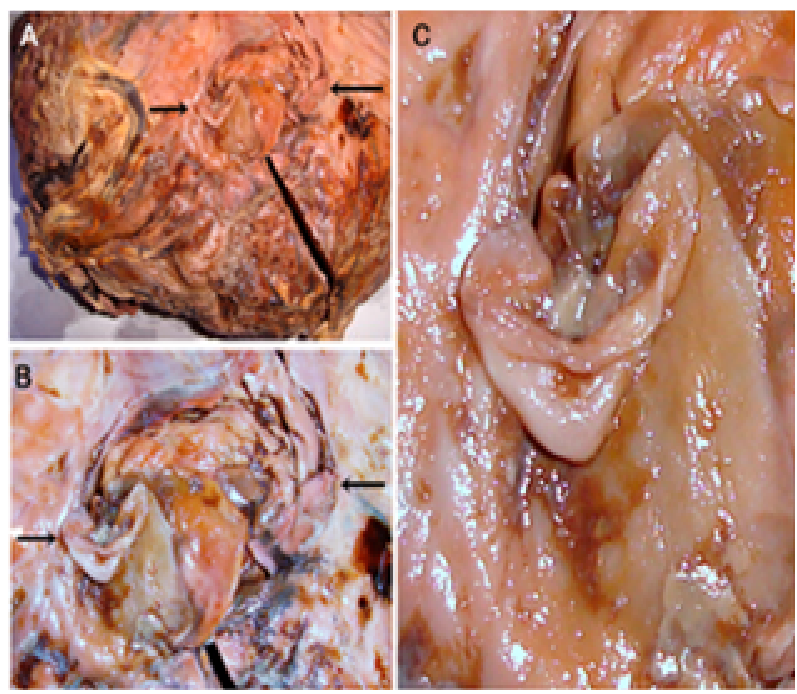

Figure 4. A: it is a panoramic view of a gross retroperitoneal tumor, a leiomyosarcoma. B: closed up of pair of embryonic bodies. Complex which are point out. C: it's a further close up of the former two.

variant scalable geometric triangular chiral hexagonal structures in interface with bio-signature patterns [20].

\section{MATERIALS AND METHODS}

Were observed and analyzed vertically and horizontally over 100 images of the shroud of Turin under different intensity of contrast and brightness, based in pattern recognition image analysis, and frequentist inference in witch any given pattern can be considered as one of an infinite sequence of possible repetitions of the same pattern in different scenarios. Among this notional set of repetitions, the identification of a specific pattern in an universal whole context, its possible only if there has been prior morphological visualization of these patterns in a cluster particular individual scenario a trough memory decoding of known parameters. Under this premise, we wonder if we can predict collision patterns on the Turin Shroud crucified man image.

\section{RESULTS}

In horizontal position by subtracting the central man that appears in the front image, we identify dark emission of central 10 - 12 spectral lines. These spectral lines evidence narrow alignment that delimiting head area and undergoes a curve, broadening at level of the lower extremities, this signify that some type of photon source energy its generate in the brain and decay slowly at distal level.

Decay spontaneous emission is the process by which a light source such as an atom, molecule, nanocrystal or nucleus in an excited state undergoes a transition to a state with a lower energy. Electromagnetic radiation 


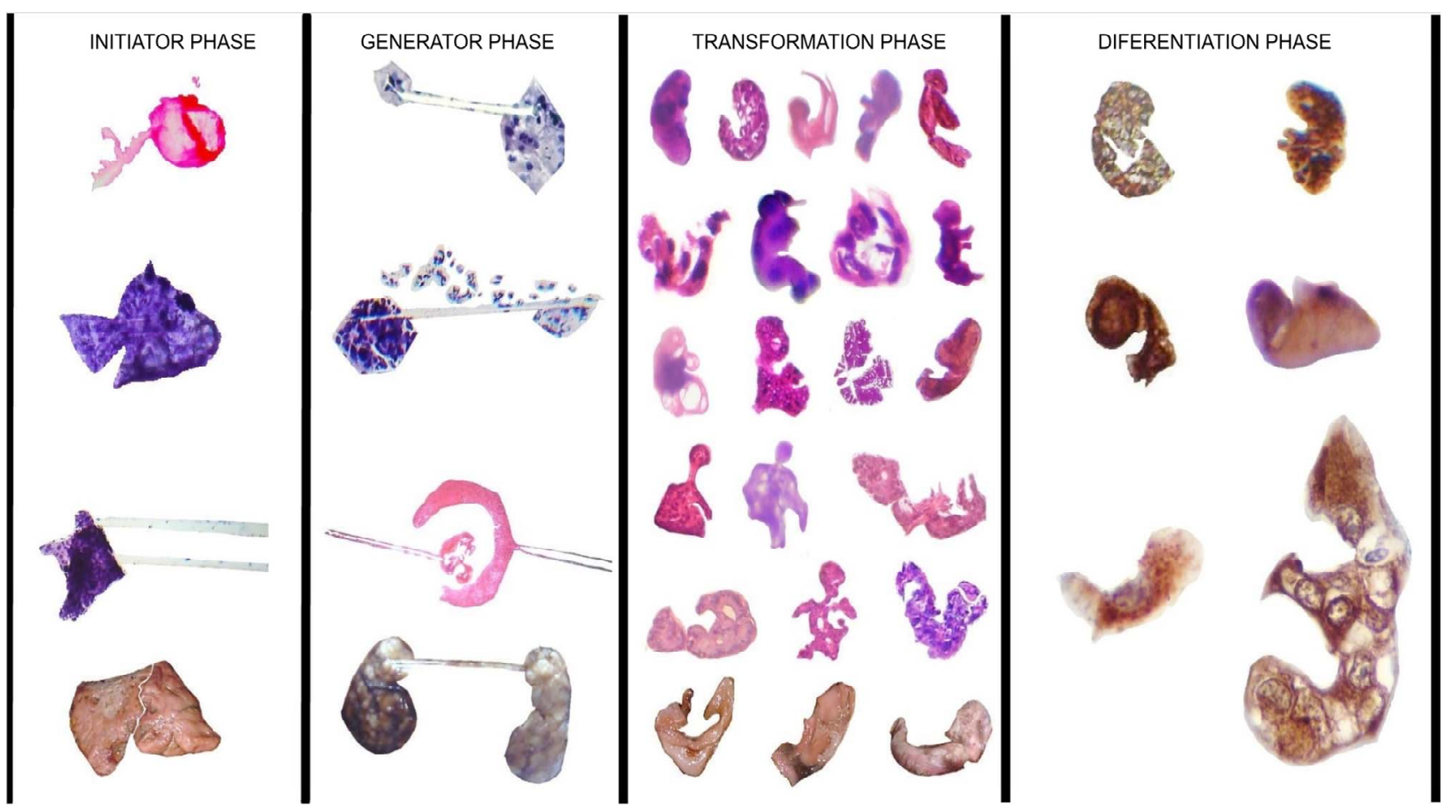

Figure 5. Sequential development of embryoid body pattern in malignant tumors and in extreme injured tissues.

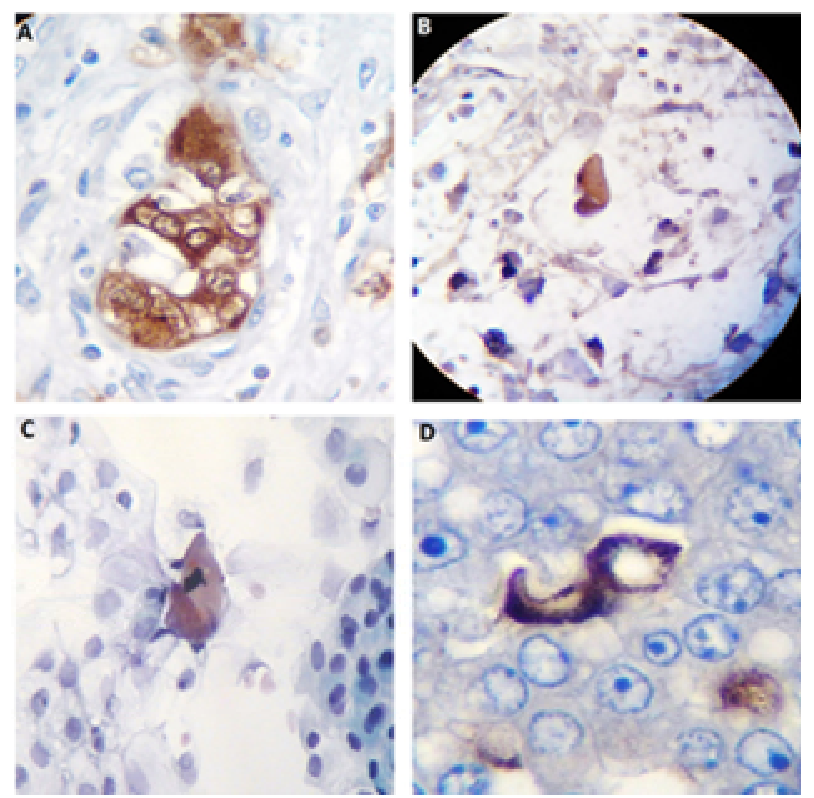

Figure 6. Neuron-specific enolase immunostaining analysis. A is a micrograph revealing high enolase immunopositivity in a well-defined self-assembled embryoid body pattern from a case of prostate carcinoma $(40 \times)$ B shows highly selective enolase immunopositivity in a self-assembled embryoid body structure inside a hexagonal geometric pattern in a case of lung carcinoma (10×); C is a micrograph revealing enolase. immunopositivity in a well-defined, self-assembled embryoid body pattern in a case of squamous cell carcinoma of the skin (10×); D reveals highly selective enolase immunopositivity in embryoid body structure from a case of esophageal squamous cell carcinoma $(40 \times)$.


Figure 7. Embryogenic bodies are not limited to cancer tissues. A and B show embryogenic bodies in damaged tissues generates by traumatic injury in patient affected by landmine. $\mathrm{C}$ shows embryogenic body in non-traumatic amputation due to complications of the vascular system (the blood vessels), in diabetes patient. D shows pancreas tissue of a patient who suffered abdominal wound gunshot.

broadening due to extended conditions may result from changes to the spectral distribution of the radiation as it traverses its path to the observer. It also may result from 
the combining of radiation from a number of regions which are far from each other. And it's a result from the velocity changing in a decay energy (Figure 8).

Outside the body man image there is no evidence of these spectral lines. The spectral lines generate a hexagon pattern, geometry that delimits the man body background (Figure 9) and structuring spatial triangular chiral mirror image organization (Figure 10). Other chiral patterns are identified in terms of helicity waves (Fi-

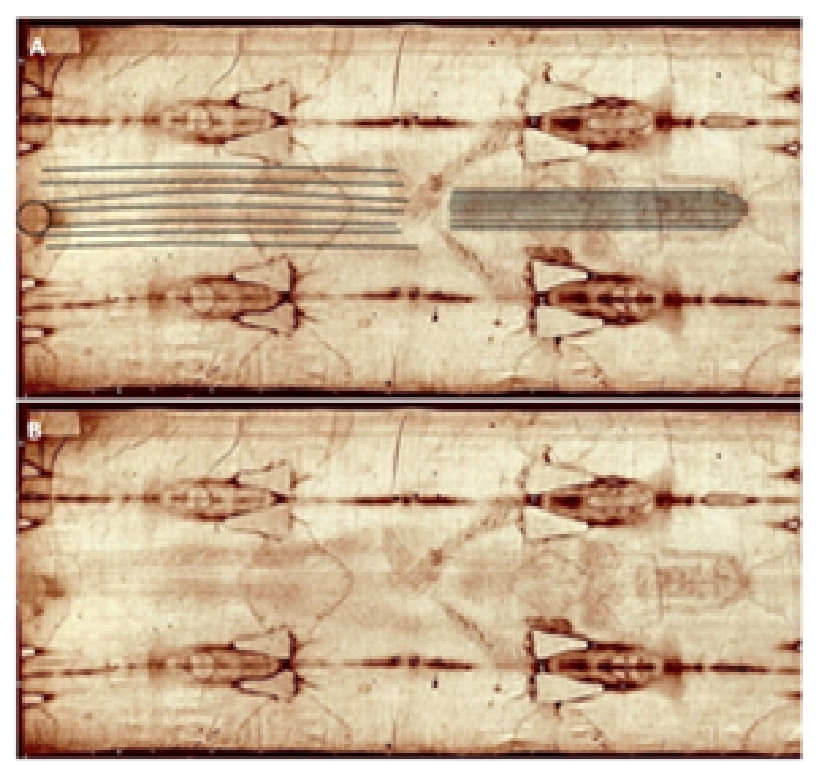

Figure 8. A It's a detachment of image B. The Shroud of Turin in horizontal position. By subtracting the central man in the front image is identified a spectral dark emission of central 10 12 lines. (C)1978 Barrie M. Schwortz Collection, STERA, Inc.

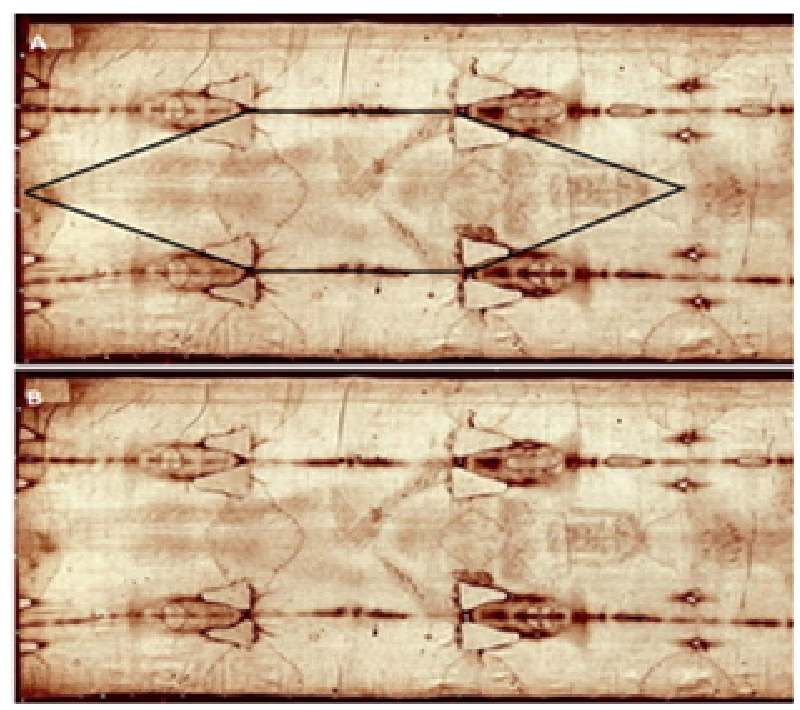

Figure 9. A: it's a detachment of image B. The spectral lines generate in the cloth a hexagon pattern strategically framed by the position of four triangular mirror images. Geometry that perfectly delimits the man body background. (C)1978 Barrie M. Schwortz Collection, STERA, Inc.

\section{gures 11 and 12).}

In vertical position, triangular chiral mirror images on each side of the midline in the front and back of the body area become evident. Draws our attention the identification of triangular mirror images at thorax level and below the knees (Figure 13), this pattern appear specifically in areas of stagnant blood, from which emerge astonishment, in the front body 8 embryonic mirror chiral bodies corresponding to 8 triangular mirror images (Figure 14).

These embryogenic structures show in detail a forked in the middle of the face that coincides with the developments of 9 weeks fetus (Figures 15-19).

Literature review makes reference to these triangular areas as holes resulted from fire damage in 1593. Real fire experiments, carried out, demonstrate the possible slanting fall of a burning metal strip or lid part. Initial
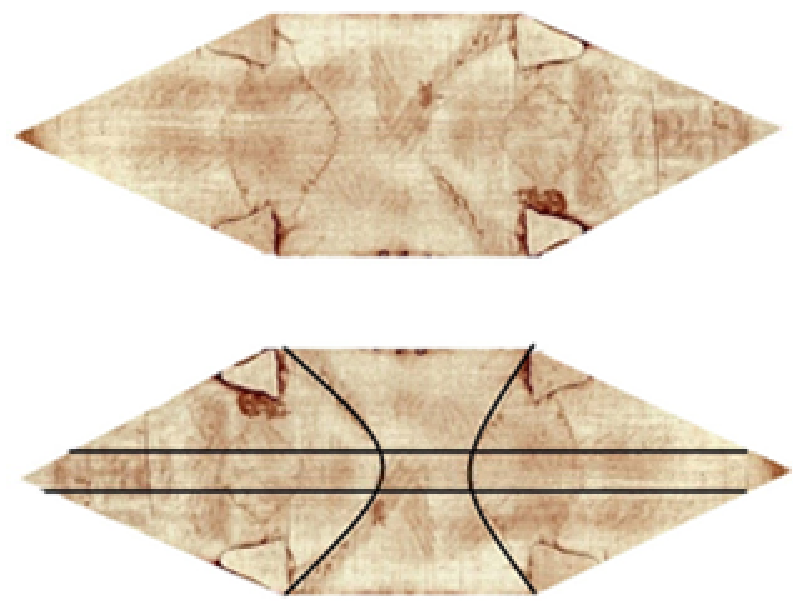

Figure 10. The hexagon geometry generates spatial substrate of triangular chiral mirror image organization in the shroud.

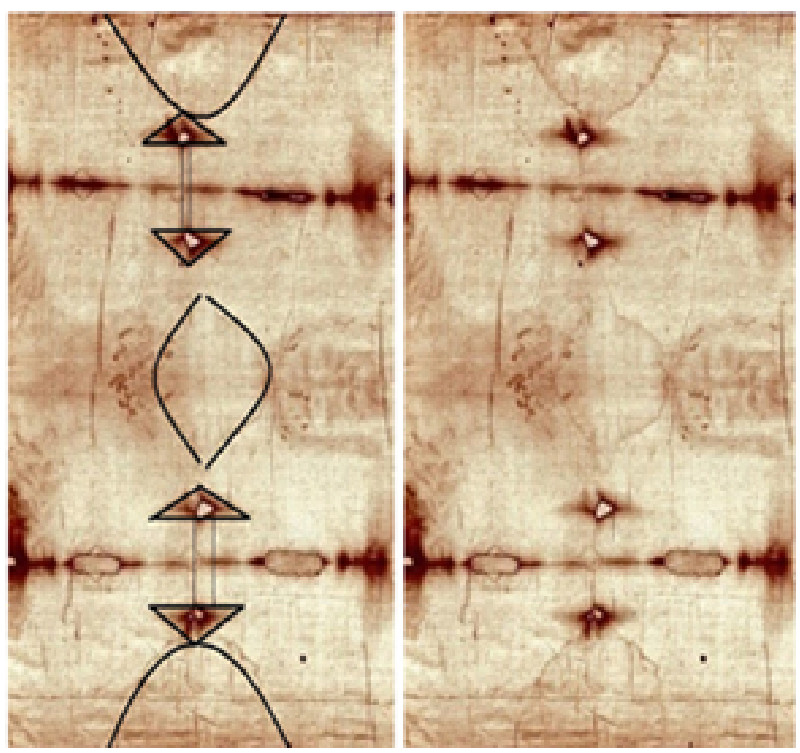

Figure 11. Electromagnetic field wave polarization described on the fabric triangular chiral mirror images pattern. 


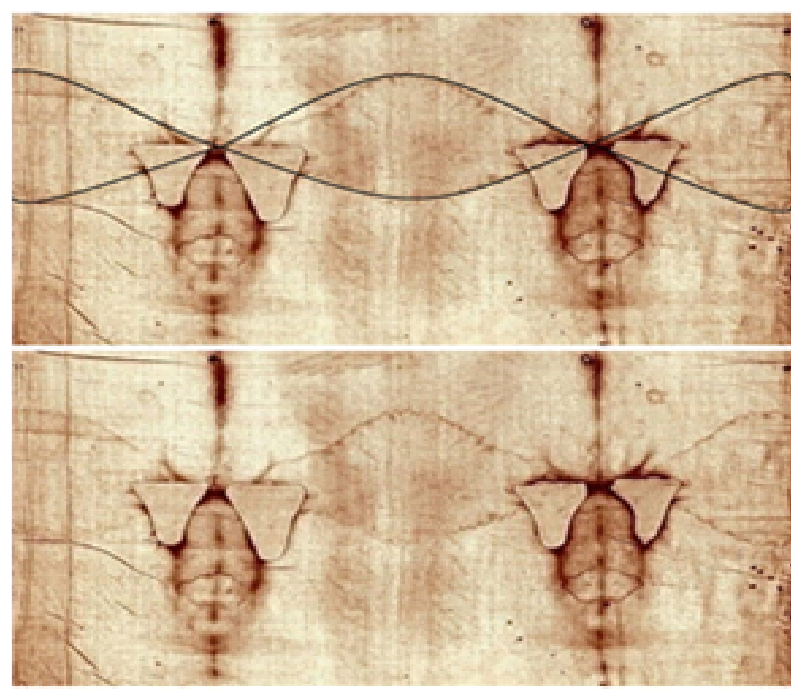

Figure 12. Electromagnetic field wave polarization described on the fabric helicity pattern.
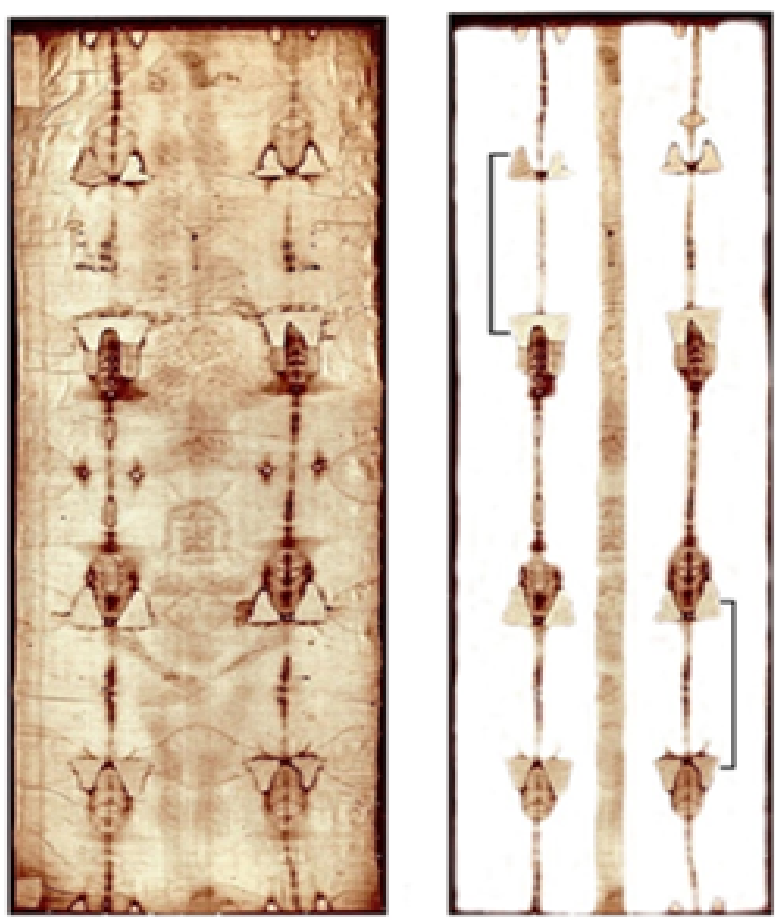

Figure 13. The Shroud of Turin front image in vertical position, becomes more evident the commanded triangular chiral mirror images on each side of the midline, in the front and back of the body area.

size of the burn hole crater which forms a rough triangle with a base of $10 \mathrm{~cm}$ and a height of $18 \mathrm{~cm}$. Researchers cannot believe it possible for it having been produced by simply a few drops of molten metal thus form, one cannot explain the reason for triangular repair patches indeed, regarding the 1st and 4th layers, each individual patch was first of all triangular in shape according to that of the burn hole it covered. A collapse of the center of the

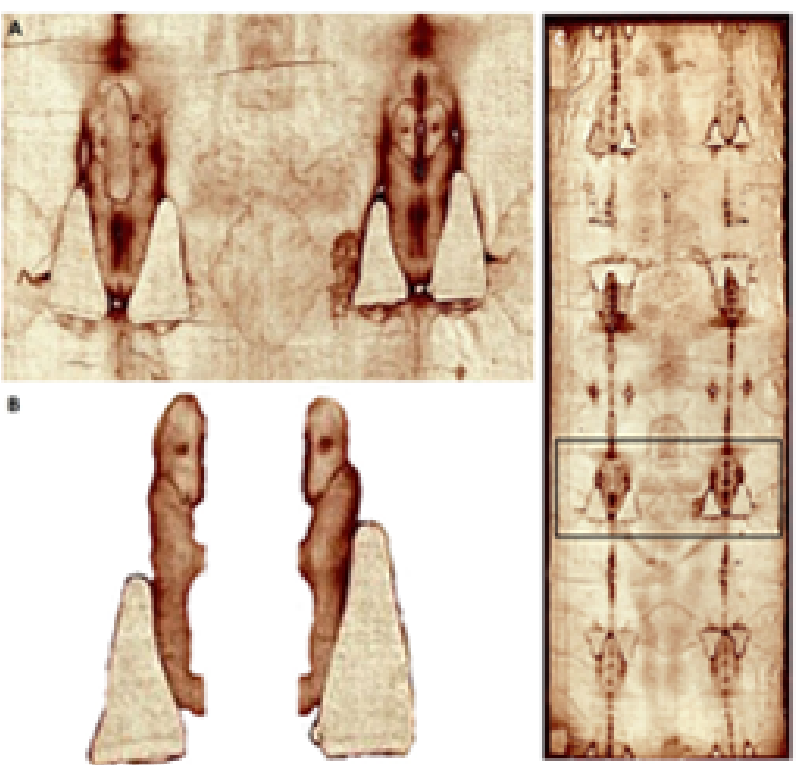

Figure 14. A. The Shroud of Turin front image at thorax level show lateral areas of stagnant blood. From which emerges 4 embryonic mirror chiral bodies corresponding to 4 triangular mirror images. B Decomposition of the principal image identifie a pair of defined embryogenic structures in interphase with pair of triangular patterns. $C$ the Shroud of Turin panoramic area where the images were documented.
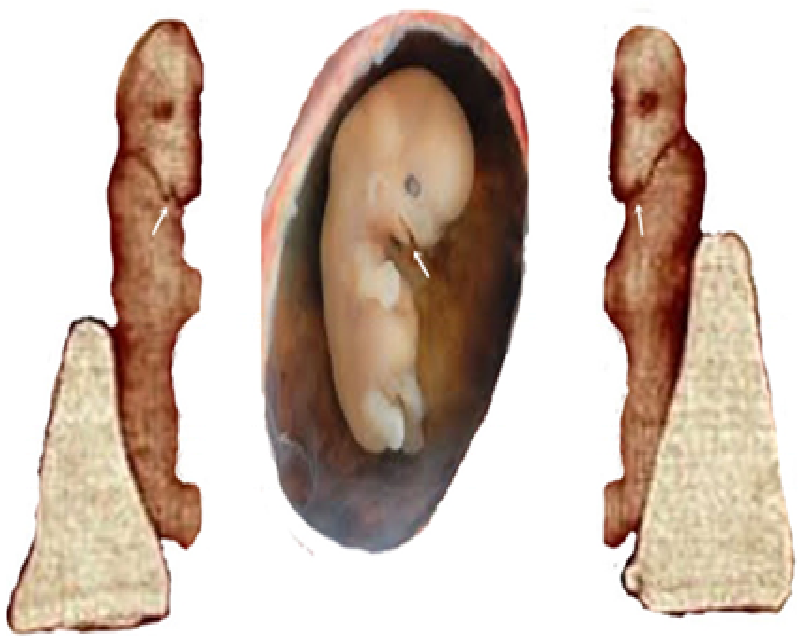

Figure 15. Shows Decomposition of the principal image that identifies a pair of defined embryonic structures in interphase with pair of triangular patterns in which was observed a forked in the middle of the face (arrow) that coincide with the develop of fetus of 9 weeks comparative image.

reliquary casket lid seems more likely, perhaps caused by the impact of a burning. What we observe here its pairs of perfect triangular chiral images in total 16 that match exactly with 16 embryogenic bodies that correspond perfectly with the areas of blood stagnation blood (Figure 20). It's not possible to replicate this triangular bio signature order by simple fire cloth penetration. Another 

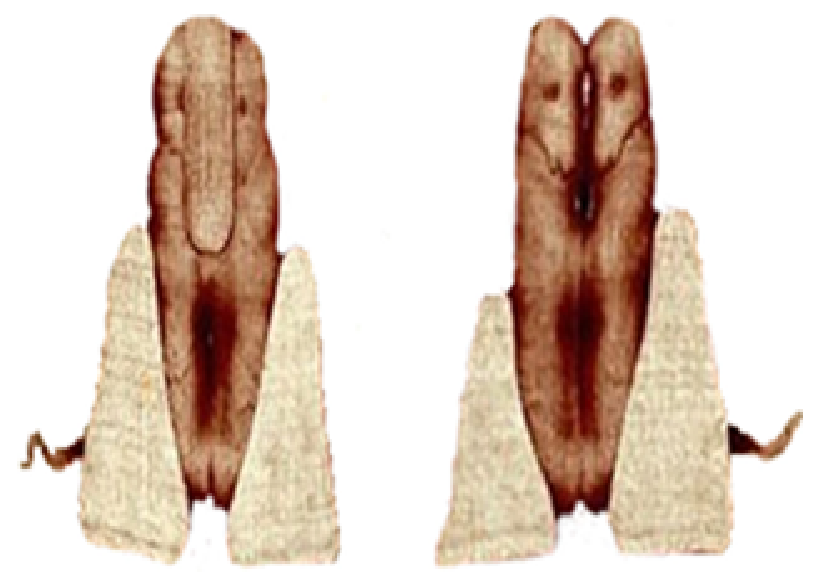

Figure 16. Decomposition image shows 4 embryogenic bodies in interface with 4 triangular geometric patterns.

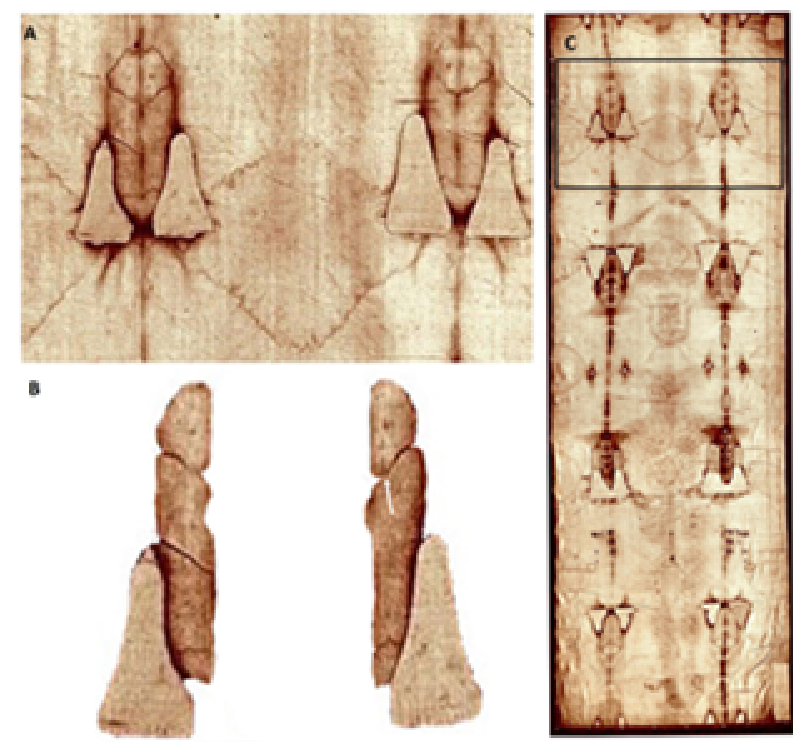

Figure 17. A, The Shroud of Turin front in inverse position at knees level show lateral areas of stagnant blood from which emerges 4 embryonic mirror chiral bodies corresponding to 4 triangular mirror images. B. Decomposition of the principal image identifies a pair of defined embryogenic structures in interphase with pair of triangular patterns $C$ The Shroud of Turin panoramic area where the images were documented.

finding that complements and endorses our observations is the identification of pair of embriogenic bodies attached by a triangular pattern in the face area not directly affected by the alleged fire (Figure 21).

Review of the literature on the Turin shroud have not reference to spectral lines, chirality, helicy geometric patterns and even less embryogenic bodies observed on their surface.

\section{DISCUSSION}

In biology, cancer is the most beautiful natural model of a chaotic system, which under uncontrolled prolife-
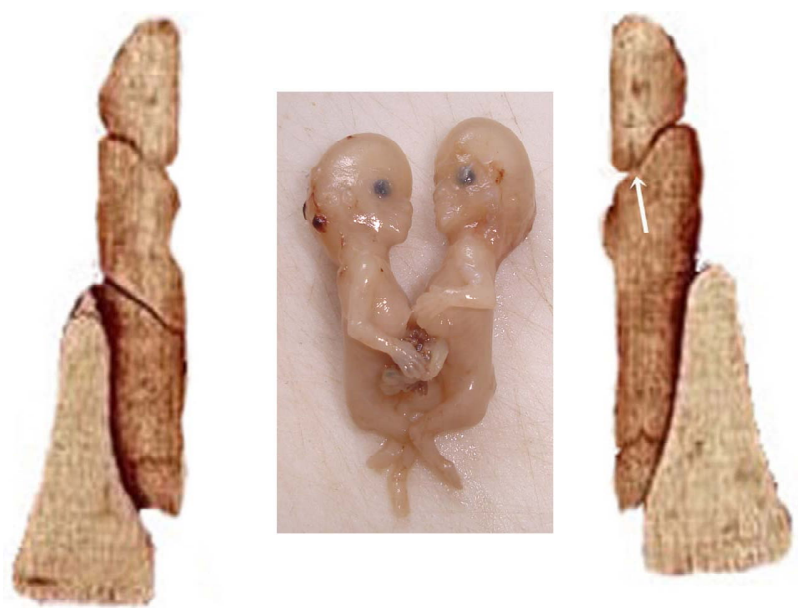

Figure 18. Shows Decomposition of the principal image that identifies a pair of defined embryonic structures in interphase with pair of triangular patterns in which was observed a forked in the middle of the face (arrow) that coincide with the develop of 6 weeks fetus.

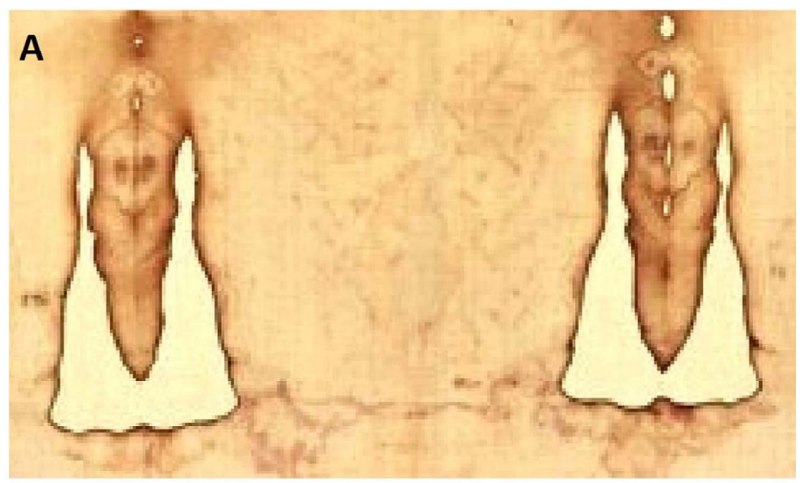

B
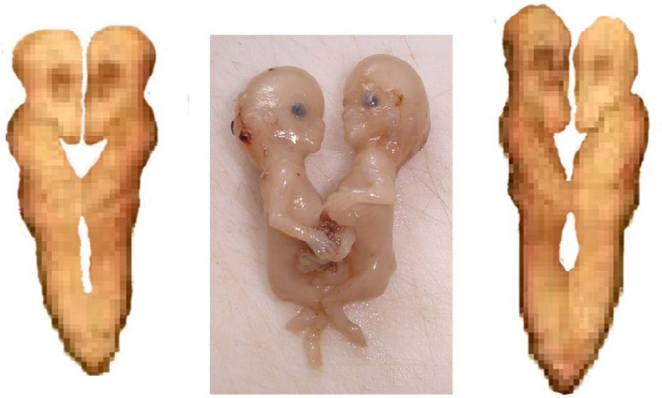

Figure 19. A. The Shroud of Turin dorsal image at back level show lateral areas of stagnant blood from which emerges 4 embryonic mirror chiral bodies corresponding to 4 triangular mirror images B. Decomposition of the principal image identifies in close up a pair of defined embryogenic structures that coincide with the develop of 6 weeks fetus.

rations, generates extreme disorder that finally causes intercellular collisions and extreme damage tissue. The authors have described and documented fractal self-assembly of geometric triangular chiral hexagonal crystallike complex organizations (GTCHC) and interface comet tail effect patterns with embryoid body reassembly 




Figure 20. A. The Shroud of Turin dorsal image in inverse position at lower extremities show lateral areas of stagnant blood from which emerges 4 embryonic mirror chiral bodies corresponding to 4 triangular mirror images $B$. Decomposition of the principal image identifies in close up a pair of defined embryogenic structures in interphase with pair of triangular patterns C. The Shroud of Turin panoramic area where the images were documented.

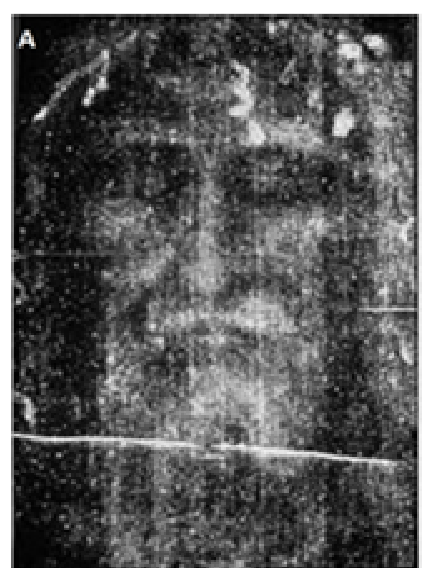

B

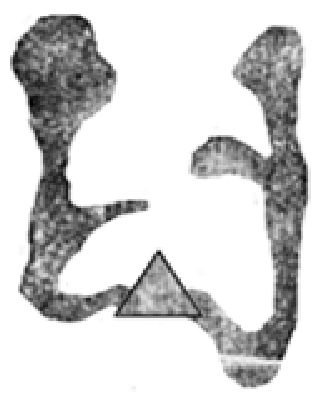

Figure 21. A Shroud of Turin face of the crucified man. B. Decomposition image identifies a pair of defined embryogenic structures attached by visible perfect triangular pattern. (C1978 Barrie M. Schwortz Collection, STERA, Inc.

transformation in cancer processes.

According to this novel observation cancer incorporates a real visualization world with a great surprising finding in biology, physics, and geology. This visualiztion platform literally allows us to see what would otherwise remain completely invisible. In a brief glance, we could recognize patterns or identify suitable features in noise data-something that is difficult or impossible to achieve with more traditional data analysis. It would have been practically impossible to achieve these findings on the Shroud of Turin, if we do not possess previous knowledge on substrate morphology to understand and decode the encrypted information hidden in this fractal geometry. Can be very difficult to understand the relationship between the cancer and the image man transfiguration in the Shroud of Turin, to facilitate an approach to this question, we should raise this concern in context of visual perception: How we can explain the identification of geometric similar patterns in dissimilar systems? The connection factor here is the extreme damage tissue that precedes the formation of these self-assembly patterns.

Pattern representation of triangular geometry in interphase with human being is not new, who built the pyramids and the sphinx in the same plane in some way knew this interrelationship between geometry and human beings (Figure 22). With a codification message: Geometric order could potentially be life-bearing.

We are confronted with irreducible geometric matrix, order noble structures that remain unchanged at the microscopic, macroscopic, megascopic levels during transit. Thus, independent of the type of collision event, collisions generate geometry in a space-time interval. In the same way, in any given place geometry has existed previously in the interaction area as a background of a collision event.

The biology of cancer collisions and the shroud docu-

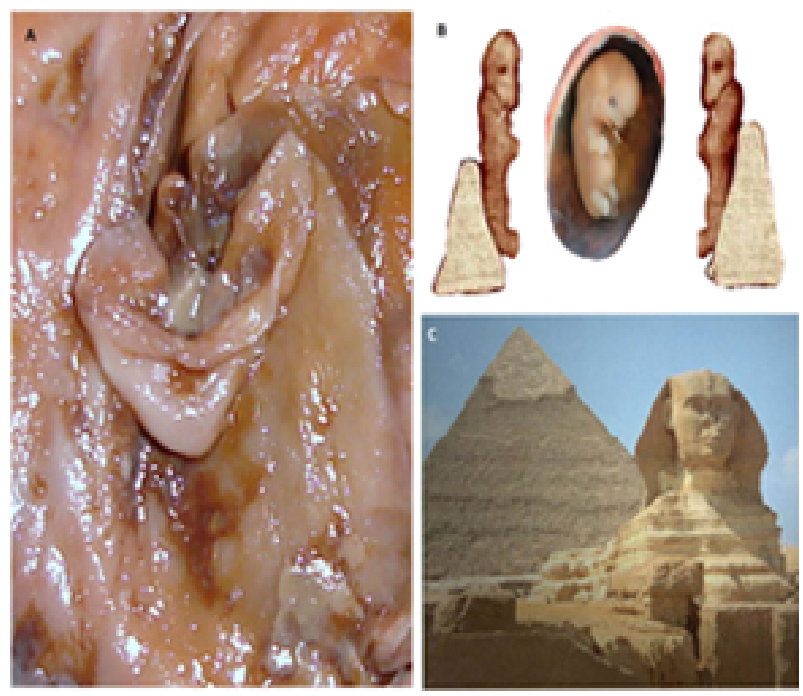

Figure 22. Comparative pattern analysis of biologic cancer and injured tissues with ancestral geometry A shows embryogenic body in interface with triangular in massive great abdominal sarcoma tumor. B shows embryogenic body in interface with triangular pattern on The Shroud of Turin C Panoramic view shows intimate positional spatial relation between Sphinx monument with Giza’s Great Pyramid. 
mented patterns of comparative image analysis are selfexplanatory, and the observable similarity is absolutely impressive. From these images that act as evidence, we can state with absolute consensus that collision events generate in the spectrum of space-time bio-signature hyper-order domain states at all scales, which corroborates the idea that the dynamic geometric order occurs through an MF activity.

The irreducible geometry and the embryogenic patterns bearing on the Shroud of Turin was generate probably with the release of electromagnetic field, the evidence its the spectral lines that exhibits a characteristic signature on a plot of the residual radiation. These spectral lines are not uniform, narrowing thin lines pattern in the proximal head area contrast with the broadening lines in distal areas, and its indication of decay energy and changes in the velocity of the molecules in the traversal trajectory. In physics Dicke narrowing is a phenomenon that dramatically reduces the Doppler width of spectral lines, due to frequent velocity-changing collisions [21, 22]. A similar phenomenon occurs for electromagnetically induced transparency resonances, and facilitates modeling the spectral line shapes with speed-dependent broadening and Dicke narrowing.

The spatial relation between the spectral lines and the magnetic abnormalities over the surface of the cloth is a direct visible proof of the strong energy release from the speed energy in the collision event. Accordingly, the geometric patterns in the cloth appear to be younger than the spectral lines, the embryogenic bodies and the man image complete the end sequential space time energy scenario and are younger than the geometric complexes, indicating that the collision surface spectral lines geometric complexes and embryonic bodies were structured in a sequential way.

The formation of embryogenic bodies its trigger by extreme body tissue damage, rupture of vessels bleeding and the loss of the spatial organizational architecture of collagen and extracellular matrix From the blood and injured tissues emerge inflammatory cytokines that activate stem cells, in principle this mechanism serve to repair process, but when this signal path way exceeds time and space, namely, the lesion is too chronic or too large, a reserve mechanism of embryonic protein its activates with cascading of interactions. The candidates are BMP and Notch proteins both of them activated by inflammatory cytokines. The name BMP was first given to three proteins purified from a demineralized bovine bone preparation that induced ectopic cartilage and endochondral bone when implanted in experimental animals [23]. these molecules regulate biological processes as diverse as cell proliferation, apoptosis, differentiation, cell-fate promote hematopoietic differentiation of human embryonic stem cells recruitment and extravasation into injured tissues and subsequent modulation of the proper morphogenesis in embryo development.

Additionally Cytokines Induce Notch Signaling proteins [24]. The Notch signaling network is an evolutionarily conserved intercellular signaling pathway that regulates interactions between physically adjacent cells, playing a role in a variety of developmental processes by controlling cell fate signaling in embryo polarity [25]. Promotes proliferative signaling during neurogenesis and its activity is inhibited by Numb to promote neural differentiation. [26] and participate in development and cancer [27]. Under the control of these signal pathway aggregates that we call, damage tissue derivate stem cells (DTDS), forming spheres, or as adherent aggregates take spatial positional polarity identity to generate biological active clonal human embryoid template.

Can we predict function from structure? Before leaping to high level functional conclusions from low level anatomy, it may be a good idea to ask what low level functions can even be inferred from these structures. Salamanders deal with injury in a remarkable way. The end result is the complete functional restoration of any tissue, on any part of the body including organs. The intimate trigger correlate patterns of embryogenic bodies with damage injured tissues make structure-function links more obvious.

These structures evoke life, regeneration not death. Representing for us the genetic expression of reversal cells process, mechanisms that in biology are intimately linked with DNA repair.

\section{CONCLUSION}

Our findings suggest that the image of a crucified man on the Shroud of Turin is a real physical electromagneticcollision event in response to extreme tissue injury, with the fact that supports our previous findings in cancer tissues as real and predictable. Further studies must be carried out to determine whether morphogenic order proteins and DNA derived from these emergent damage tissue derivate stem cells (DTDS), functioning as natural autolog genetic material, could be used to design biologic templates in regenerative medicine and develop novel strategies in cancer therapy.

\section{ACKNOWLEDGEMENTS}

The author thanks Dr. Juan Carlos Triana and Dr. Jorge Ovidio Cruz, executive directors of the Hospital Department of Villavicencio and Hospital Department of Granada, for their financial support of this research and help with logistics as well as the medical student Jennifer Catherine Camacho for the picture work and panel arrangement. Special thanks are due to Barrie Schwortz, Documenting Photographer of the Shroud of Turin Research Project (STURP) at the 1978 exposition, 
for permission to use his copyrighted images.

\section{REFERENCES}

[1] Damon, P.E. et al. (1989) Radiocarbon dating of the Shroud of Turin. Nature, 337, 611-615. http://dx.doi.org/10.1038/337611a0

[2] Ball, P. (2008) Material witness: Shrouded in mystery. Nature Materials, 7, 349-350. http://dx.doi.org/10.1038/nmat2170

[3] Pellicori, S.F. (1980) Spectral properties of the Shroud of Turin. Applied Optics, 19, 1913-1920. http://dx.doi.org/10.1364/AO.19.001913

[4] Carter, G.F. (1984) Formation of the image on the Shroud of Turin by x-rays: A new hypothesis. Archaeological Chemistry, 425-446.

[5] Miller, V.D. and Pellicori, S.F. (1981) Ultraviolet fluorescence photography of the Shroud of Turin. Journal of Biological Photography, 3, 71-85.

[6] Rogers, N. and Arnoldi, A. (200) Scientific method applied to the Shroud of Turin. University of California Los Alamos National Laboratory Los Alamos. http://www.shroud.com/pdfs/rogers2.pdf

[7] Morris et al. (1980) X-ray fluorescence investigation of the Shroud of Turin. X-Ray Spectrometry, 9, 40-47. http://dx.doi.org/10.1002/xrs.1300090203

[8] G. Fanti and M. Moroni (2002) Comparison of luminance between face of turin shroud man and experimental results. The Journal of Imaging Science and Technology, 46, 142-154.

[9] Adler, A.D. and Whanger, A. (1997) Concerning the side strip on the Shroud of Turin. http://www.shroud.com/adler2.htm

[10] Mills, A. (1995) Image formation on the Shroud of Turin. Interdisciplinary Science Reviews, 4, 319-326. http://dx.doi.org/10.1179/030801895794105406

[11] Fanti, G., Lattarulo, F. and Scheuermann, O. (2005) Body image formation hypotheses based on corona discharge. http://www.dim.unipd.it/fanti/corona.pdf

[12] Maggiolo, F. The double superficiality of the frontal image of the Turin Shroud. Journal of Optics, 6, 491-503. http://dx.doi.org/10.1088/1464-4258/6/6/001

[13] Heller, J.H. and Adler, A.D. (1980) Blood on the Shroud of Turin. Applied Optics, 19, 2742-2744. http://dx.doi.org/10.1364/AO.19.002742

[14] Heller, J.H. and Adler, A.D. (1981) A chemical investigation of the Shroud of Turin. Canadian Forensic Society Scientific Journal, 14, 81-103.

[15] Bollone, P.I. and Gaglio, A. (1984) Demonstration of blood, aloes and myrrh on the Holy Shroud with immunofluore- scence techniques. Shroud Spectrum International, 13, 38.

[16] Diaz, J., Jaramillo, N. and Murillo, M. (2007) Geometric triangular chiral hexagon crystal-like complexes organization in pathological tissues biological collision order. PLOS ONE, 2, e1282.

[17] Diaz, J.M. and Murillo (2009) Framework of collagen type I vasoactive vessels structuring invariant geometric attractor in cancer tissues: Insight into biological magnetic fields. PLOS ONE, 4, e4506. http://dx.doi.org/10.1371/journal.pone.0004506

[18] Diaz, J.M. and Murillo, B.A. (2011) Intercellular cancer collisions generate an ejected crystal comet tail effect with fractal interface embryoid body reassembly transformation. Cancer Management and Research, 3, 143-155. http://dx.doi.org/10.2147/CMAR.S17402

[19] Diaz, J.M. and Murillo (2012) Phenotype characterization of embryoid body structures generated by a crystal comet effect tail in an intercellular cancer collision scenario. Cancer Management and Research, 4, 9-21. http://dx.doi.org/10.2147/CMAR.S25810

[20] Diaz, J. (2013) Electromagnetic field released in collision impact events generate in the matrix interface fractal scalable invariant geometric triangular chiral hexagonal structures. Open Journal of Geology, 3, 187-200. http://dx.doi.org/10.4236/ojg.2013.33022

[21] Dicke, R.H. (1953) The effect of collisions upon the doppler width of spectral lines. Physical Reviews, 89, 472473. http://dx.doi.org/10.1103/PhysRev.89.472

[22] Kristensen, D.M., Kalisz, M. and Nielsen, J.H. (2005) Cytokine signalling in embryonic stem cells. Acta Pathologica, Microbiologica et Inmunologica Scandinava, 113, 11-12.

[23] Wozney et al. (1988) Novel regulators of bone formation: Molecular clones and activities. Science, 242, 1528-1534.

[24] Varnum-Finney et al. (2000) Pluripotent, cytokine-dependent, hematopoietic stem cells are immortalized by constitutive Notch1 signaling. Nature Medicine, 11, 12781281. http://dx.doi.org/10.1038/nature02040

[25] Spyros et al. (1999) Notch signaling: Cell fate control and signal integration in development (Review). Science, 284, 770-776.

[26] Gaiano, N.G. and Fishell (2002) The role of notch in promoting glial and neural stem cell fates. Annual Review of Neuroscience, 25, 471-490.

[27] Bolós, V., Grego-Bessa, J. and de la Pompa, J.L. (2007) Notch signaling in development and cancer. Endocrine Reviews, 28, 339-344.

http://dx.doi.org/10.1210/er.2006-0046 